\title{
Interactions between insulin resistance and insulin secretion in the development of glucose intolerance
}

\author{
Melissa K. Cavaghan, ${ }^{1}$ David A. Ehrmann, ${ }^{1}$ and Kenneth S. Polonsky ${ }^{2}$ \\ ${ }^{1}$ Department of Medicine, The University of Chicago, Pritzker School of Medicine, Chicago, Illinois, USA \\ ${ }^{2}$ Department of Medicine, Washington University School of Medicine, St. Louis, Missouri, USA \\ Address correspondence to: Kenneth S. Polonsky, Department of Medicine, Washington University School \\ of Medicine, Campus Box 8066, 660 S. Euclid Avenue, St. Louis, Missouri 63110-1093, USA. \\ Phone: (314) 362-8060; Fax: (314) 362-8015; E-mail: polonsky@im.wustl.edu.
}

Subjects who develop type 2 diabetes have a complex phenotype with defects in insulin secretion, increased hepatic glucose production, and resistance to the action of insulin, all of which contribute to the development of overt hyperglycemia. Although the precise mechanisms whereby these three factors interact to produce glucose intolerance and diabetes are uncertain, it has been suggested that the final common pathway responsible for the development of type 2 diabetes is the failure of the pancreatic $\beta$ cell to compensate for insulin resistance (1). Here, we review evidence for this model from both human and animal studies, and we consider the effects of specific drug treatments and of heightened FFA levels on insulin sensitivity and insulin secretion in individuals at risk for type 2 diabetes.

Coleman and coworkers (2), drawing on their work with the $o b / o b$ mouse, first proposed that a failure of $\beta$ cell compensation for insulin resistance is responsible for the development of type 2 diabetes. When insulinresistant $o b / o b$ mice, which provide a genetic model of obesity, were bred with mice of different strains, the phenotypes of the progeny differed depending on their genetic background. Mice carrying the $o b$ mutation in the C57BL/ 6 background had relatively normal blood glucose levels, whereas in the C57Ks background the animals developed overt hyperglycemia. The major difference between these two mouse strains lay in their $\beta$ cell responses. The nondiabetic C57BL/ 6 mice underwent a marked expansion of $\beta$-cell mass, whereas C57Ks animals showed histologic evidence of islet degeneration, degranulation, and progressive atrophy. The C57Ks animals accordingly had insulin levels about one-tenth those of the C57BL/6 animals, and these levels declined progressively with age. These data indicated that the genetic background on which insulin resistance develops influences the adequacy of pancreatic $\beta$-cell compensation for insulin resistance, and hence the predisposition to diabetes.

$\beta$-cell responses to insulin resistance

In the setting of insulin resistance and obesity, insulin levels typically increase to maintain normal glucose tolerance. Insulin secretion rates are three to four times higher in obese insulin resistant subjects than in lean controls (3). The response of the $\beta$ cell to glucose in the setting of insulin resistance has been studied by administering graded intravenous glucose infusions to insulin-sensitive and insulin-resistant subjects with normal glucose tolerance (4). Figure 1 depicts insulin and insulin secretion rates at each level of plasma glucose achieved. This representation of the data allows $\beta$-cell responsiveness to glucose to be examined independently of differences in glucose levels. As this figure shows, insulin levels and insulin secretion rates are clearly increased in insulin-resistant subjects. Further work showed that this hyperinsulinemia results from both increased secretion and reduced clearance of insulin. The upward and leftward shift seen in the dose-response relationship between glucose and insulin secretion in insulin-resistant subjects (Figure 1) represents an adaptive response of $\beta$ cells that helps to maintain normal glucose tolerance. Kahn and colleagues (5) likewise detected an inverse relationship between insulin sensitivity and insulin secretion, demonstrating $\beta$-cell compensation for insulin resistance. Thus, as insulin resistance increases, first-phase insulin release increases proportionately to maintain normal glucose tolerance.

This compensatory hypersecretion of insulin reflects not only expansion of $\beta$-cell mass, but also altered expression of key enzymes of $\beta$-cell glucose metabolism. Evidence for both of these mechanisms comes from the Zucker fatty rat, which is obese and insulinresistant but which enjoys adequate $\beta$-cell compensation and does not develop diabetes. In contrast, the Zucker diabetic fatty (ZDF) rat is obese and insulinresistant and develops overt hyperglycemia. At 6 weeks of age, prior to the onset of diabetes, $\beta$-cell mass is increased twofold in the ZDF rats compared with lean controls and is similar to that found in Zucker fatty rats. Between 6 and 12 weeks of age, however, $\beta$-cell mass increases about fourfold in the Zucker fatty rats but only about twofold in the ZDF rats (6). Thus, the development of overt diabetes in the ZDF rat is associ- 


\section{Figure 1}

Plasma insulin concentrations (a) and insulin secretion rates $(\mathbf{b})$ in response to molar increments in the plasma glucose concentration during a graded glucose infusion in insulin-resistant (dashed line) and insulin-sensitive (solid line) groups. Reprinted with permission (4). a

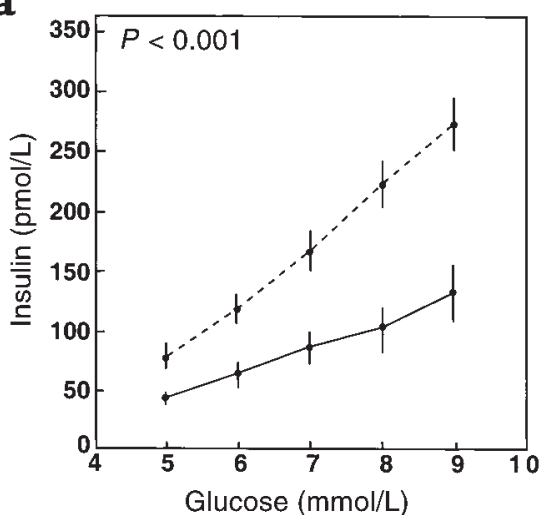

b

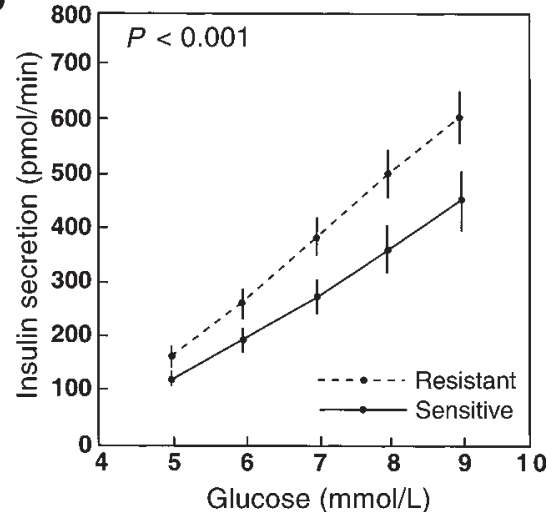

ated with a failure of adequate $\beta$-cell mass expansion in the face of insulin resistance. When the hypersecretion of insulin observed in perfused pancreata in ZDF and Zucker fatty rats is corrected for the increase in $\beta$-cell mass, insulin secretion rates are seen to be lower than those observed in lean control animals (6), suggesting that the increase in $\beta$-cell mass represents a functionally significant compensation. Estimated rates of $\beta$-cell proliferation, based on bromodeoxyuridine incorporation, suggest that the failure of $\beta$-cell mass to expand adequately in the ZDF rat is due not to a failure of $\beta$ cell proliferation but to an enhanced rate of $\beta$-cell death, presumably resulting from apoptosis.

Change in the relative activities of the two glucosephosphorylating enzymes glucokinase (GK) and hexokinase (HK) also contributes to insulin hypersecretion in insulin-resistant states. Conversion of glucose to glucose-6-phosphate is normally mediated predominantly by GK. This first, rate-limiting step in $\beta$-cell glucose metabolism represents the $\beta$ cell's glucose sensor. $\beta$-cell sensitivity to glucose and, therefore, the normal glucose-insulin secretion dose-response curve are determined predominantly by the $K_{\mathrm{m}}$ of $\mathrm{GK}$ for glucose, which is approximately $8 \mathrm{mM}$. Insulin resistance is associated with an increase in the expression of $\beta$-cell $\mathrm{HK}$, an enzyme with a significantly lower $K_{\mathrm{m}}$ for glucose. Milburn et al. noted that the hypersecretion of insulin by islets from insulin-resistant Zucker fatty rats is accompanied by increased low- $K_{\mathrm{m}}$ glucose metabolism, implicating upregulation of HK in this progression (7). Subsequently, Becker and colleagues (8) confirmed that experimental overexpression of $\mathrm{HK}$ in isolated islets induces low- $K_{\mathrm{m}}$ glucose usage and insulin release at lower glucose concentration. Although overexpression of either GK or HK can enhance glucose phosphorylation, HK causes substantially more glucose-stimulated insulin secretion and overall glucose metabolism by the $\beta$-cell than does overexpression of GK (9). Cockburn et al. compared relative $\mathrm{HK}$ and $\mathrm{GK}$ activities in islets from Zucker fatty rats and lean control animals and showed that HK but not GK activity was enhanced (10). Since this induction correlated with increased basal insulin secretion rates, it appears that the selective upregulation of HK in obesity, like the increase in $\beta$-cell mass, plays an important role in the compensatory hypersecretion of insulin observed in insulin resistance.

\section{Altered gene expression in the evolution of glucose intolerance}

Patterns of gene expression are clearly affected by the progression to overt diabetes in the ZDF rat. Tokuyama and colleagues (11) followed the expression of 30 mRNA species before and after the onset of disease and noted changes in expression levels of insulin, glycolytic enzymes, and ion channels among other gene products. Prediabetic animals showed high levels of glucose-6-phosphatase and 12-lipoxygenase expression but lower mRNA levels for GK, glycerol-3-phosphate dehydrogenase, $\mathrm{Ca}^{2+}$-ATPase, voltage-dependent $\mathrm{Ca}^{2+}$ and $\mathrm{K}^{+}$channels, and transcription factor Islet-1, relative to control animals. In diabetic animals, these changes were exaggerated and involved more genes, including reduction in insulin and GLUT2 mRNA.

This profile of pancreatic $\beta$-cell gene expression did not provide significant insight into the mechanisms of $\beta$-cell dysfunction, but the alternative approach of modifying the pancreatic gene expression experimentally has indeed yielded surprising results. See Kadowaki (12) in this Perspective series for details on these studies. Mice carrying targeted disruption of genes for the insulin receptor substrates (IRS-1 and -2) have shown distinct roles for each of these molecules. Mice lacking IRS-1 exhibit growth retardation and mild insulin resistance, but they resist diabetes because they increase their $\beta$-cell mass and induce compensatory hyperinsulinemia (12). Mice lacking IRS-2, on the other hand, exhibit mild growth retardation, insulin resistance, and early development of diabetes due to impaired proliferation of $\beta$ cell mass (13). Further evidence that $\beta$ cells, per se, are affected by these mutations derives from work with animals lacking these signaling molecules specifically in this cell type. Thus, genes for the insulin receptor, IRS- 
1 , and IRS-2 have been selectively knocked out in the $\beta$ cell (14-16). When the insulin receptor is selectively lost in $\beta$ cells (in the so-called $\beta$ IRKO mouse), $\beta$ cells lose sensitivity to glucose, leading to a progressive decline in glucose tolerance (15). Ablation of $\beta$-cell IRS- 1 results in loss of insulin secretion in response to either glucose or arginine (16). Since insulin appears to positively regulate its own gene's transcription $(17,18)$, the loss of autocrine signaling in the $\beta$ cell further reduces $\beta$-cell insulin content and insulin release (18).

\section{Human insulin resistance in normal and pathological states}

Clinical studies in humans confirm the model suggested by the animal data: that $\beta$-cell function initially compensates for insulin resistance, but that early defects in $\beta$-cell function emerge as glucose tolerance deteriorates, so that by the time diabetes is evident, $\beta$-cell function is markedly abnormal. Evidence for $\beta$-cell compensation to maintain glucose tolerance (the upward and leftward shift in the glucose-insulin secretion dose-response curve shown in Figure 1) is also seen during normal pregnancy. In the third trimester, pregnant women develop severe insulin resistance but compensate by a threefold increase in first- and second-phase insulin secretion (19). Very early alterations in $\beta$-cell function can often be demonstrated in subjects with normal glucose tolerance who are at high risk for diabetes. For example, normal glucose-tolerant women with a history of gestational diabetes exhibit reduced first-phase insulin responses but normal insulin secretory responses to oscillatory glucose infusion (20). By the time impaired glucose tolerance (IGT) has developed, defects in $\beta$-cell function can be demonstrated consistently (Figure 2) (21). The glucose-insulin secretion doseresponse relationship is flattened and shifted to the right, first-phase insulin responses during intravenous glucose tolerance testing are consistently decreased in relationship to ambient insulin sensitivity, and there is a loss of coordinated insulin secretory responses during oscillatory glucose infusion (22). Once diabetes has developed, first-phase insulin responses to glucose are virtually absent, further flattening of the glucose-insulin secretion dose-response curve ensues, and coordination of insulin secretory responses during oscillatory glucose infusion is all but lost (21).

Insulin resistance, obesity, and diabetes are also common in women with polycystic ovary syndrome (PCOS), providing another condition in which to study the interaction between insulin resistance and $\beta$ cell dysfunction. We and others have found that $\beta$-cell secretory dysfunction is common in PCOS. Incremental insulin secretory responses to meals are markedly reduced, resulting from a reduction in the relative amplitude of meal-related secretory pulses, a striking pattern to observe in nondiabetic individuals (23). Impairment of first-phase insulin secretion analyzed in relation to the degree of insulin resistance is particularly marked in women with PCOS who have a firstdegree relative with type 2 diabetes. Insulin secretory responses to a graded increase in plasma glucose (expressed in relation to insulin resistance) are also impaired in these women, as is the ability of their $\beta$ cells to respond to induced oscillations in the plasma glucose level (24). Thus, the risk imparted by insulin resistance to the development of type 2 diabetes in PCOS is enhanced by defects in insulin secretion. Further, a history of type 2 diabetes in a first-degree relative defines a subset of PCOS subjects with the most profound defects in $\beta$-cell function. Taken together, these findings are in accord with studies showing a high degree of heritability of $\beta$-cell function, particularly when examined in relation to insulin sensitivity, among nondiabetic members of familial type 2 diabetic kindreds. For more on the genetics of insulin resistance, see Stern (25) in this Perspective series.

\section{Changes in $\beta$-cell function in response to manipulation of insulin resistance}

Although $\beta$-cell dysfunction clearly occurs before the onset of overt diabetes, abnormal $\beta$-cell function in individuals at risk for diabetes but with normal glucose levels has proved more difficult to demonstrate. One approach to this problem has been to administer agents such as glucocorticoids, thiazolidinediones, or FFAs, which either exacerbate or ameliorate insulin resistance. Such treatments are intended to reveal latent, subclinical defects in $\beta$ cell function and to identify individuals at high risk of developing diabetes.

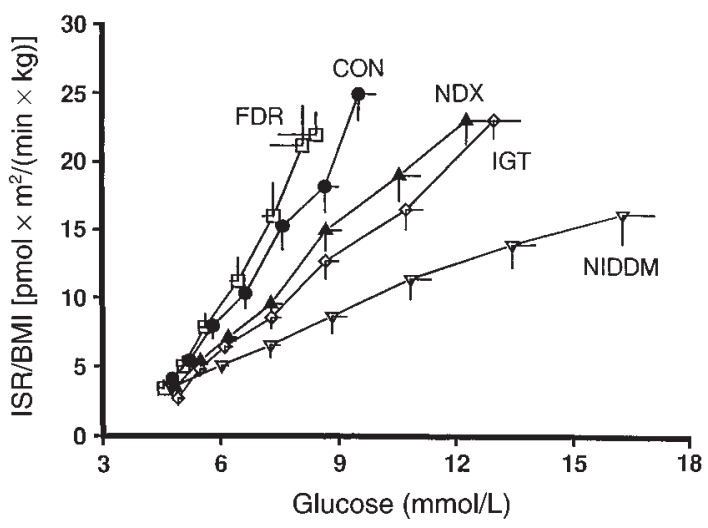

Figure 2

Dose-response relationships between glucose and insulin secretory rate (ISR) after overnight fast in control subjects (CON; filled circles), normoglycemic subjects with family history of non-insulin-dependent diabetes mellitus (FDR, first-degree relative; open squares), subjects with nondiagnostic oral glucose tolerance test (NDX; filled triangles), subjects with impaired glucose tolerance (IGT; open diamonds), and subjects with non-insulin-dependent diabetes mellitus (NIDDM; open triangles). BMI, body mass index. Reprinted with permission (21). 
The development of transient diabetes in previously nondiabetic individuals treated with short-term glucocorticoids ("steroid diabetes") has been recognized for many years. Fajans and Conn (26) found that when normal glucose-tolerant individuals with a first-degree relative with diabetes received small doses of cortisone acetate, oral glucose tolerance testing revealed that $24 \%$ had IGT and $19 \%$ had diabetes. In contrast, among those without a family history of diabetes, only $3 \%$ developed IGT and $2 \%$ developed diabetes. These abnormal responses to cortisone acetate predicted subsequent development of diabetes. Over 7 years of follow-up, the development of glucose intolerance was 17 times more common in those with initially abnormal responses. Henriksen et al. showed that nondiabetic first-degree relatives of type 2 diabetics with evidence of mild alteration of $\beta$ cell function at base line are unable to enhance their $\beta$-cell responses to dexamethasone-induced insulin resistance (27). We have recently found that low-dose dexamethasone produced significant elevations in plasma glucose and reductions in insulin sensitivity when given to either PCOS or control subjects with normal glucose tolerance. However, the $\beta$-cell response to the dexamethasone-induced rise in plasma glucose was substantially attenuated in PCOS individuals, particularly when expressed in relation to the prevailing level of glucose during an oral glucose tolerance test or the level of insulin sensitivity during a graded glucose infusion study (D.A. Ehrmann et al., unpublished data). These results suggest that women with PCOS operate near their maximal ability to secrete insulin in response to glucose and that further reductions in insulin sensitivity are met with inadequate compensation in insulin secretion. Thus, low-dose dexamethasone administration can reveal latent defects in $\beta$-cell function and identify those PCOS subjects at greatest risk for subsequent development of diabetes.

We have also used responses to the insulin-sensitizing drugs, the thiazolidinediones, to determine whether attenuation of insulin resistance would result in improved $\beta$-cell function in subjects with IGT. Detailed measures of $\beta$-cell function showed a significant increase in insulin sensitivity after daily treatment with troglitazone $(28,29)$. When $\beta$-cell function was evaluated in light of the improvement in insulin sensitivity, there were marked improvements in the glucose-insulin secretion doseresponse relationship and the ability of the $\beta$ cell to detect and to respond appropriately to changes in the plasma glucose level produced by an oscillatory glucose infusion, indicating an improvement in the glucose-sensing ability of the $\beta$ cell. Hence, deterioration of $\beta$-cell function is not irreversible in absolute terms but responds to alterations in the prevailing metabolic environment.

\section{Roles for free fatty acids in the progression of insulin resistance}

Experimental elevations of FFAs influence both insulin action and insulin secretion. Boden and coworkers have demonstrated that during infusion of a triglyceride emulsion into lean subjects, insulin resistance develops within approximately 4 hours and is associated with suppression of oxidative and nonoxidative glucose disposal (30) and increased hepatic glucose output (31). The changes in intracellular metabolism underlying these observations are complex and incompletely understood, but Shulman and coworkers have offered a model of insulin action resulting from reduced uptake or transport of glucose across the muscle membrane (this Perspective series, ref. 32).

The effects of FFAs on insulin secretion are more controversial. In vitro, FFAs acutely enhance insulin secretion $(33,34)$ and play a critical role in maintaining the insulin secretory response to glucose after a prolonged fast (35). Prolonged exposure to elevated concentrations of FFAs, however, impairs insulin secretory responses to glucose, particularly in islets from rodents predisposed to diabetes $(34,36)$. Lewis and coworkers recently demonstrated that insulin secretion during a graded glucose infusion fails to compensate for increased insulin resistance induced in healthy humans by a 48-hour elevation in FFAs (37). Similarly, we recently showed that, in the setting of increased FFAs, $\beta$-cell compensation for insulin resistance is incomplete when first-phase insulin responses are normalized for the simultaneous decrease in insulin sensitivity (M.K. Cavaghan et al., unpublished data). We also found that a 16-hour elevation of FFAs during an oscillatory glucose infusion protocol could induce, in obese, glucose-tolerant subjects, qualitative defects in $\beta$-cell function that are usually only seen in subjects with IGT (38). These observations demonstrate that the role of fat in obesity and diabetes extends beyond the ability of FFAs to induce or exacerbate insulin resistance. They suggest that, by impairing the ability of the $\beta$ cell to compensate for insulin resistance, FFAs contribute directly to the deterioration of $\beta$-cell function that accompanies the development of diabetes.

\section{Conclusions}

The studies summarized above show that there is a dynamic relationship between insulin resistance and a compensatory increase in $\beta$-cell mass and $\beta$-cell glucose metabolism. When the compensatory process is adequate, normal glucose tolerance is maintained. When $\beta$ cell compensation fails, glucose levels rise, leading to either IGT or overt diabetes. Our understanding of the mechanisms responsible for these compensatory processes remains incomplete at the molecular level. However, in view of the central role played by enhanced $\beta$-cell function in maintaining normal glucose tolerance 
in the face of insulin resistance, a more detailed understanding of these processes will likely lead to novel approaches to the prevention and treatment of diabetes.

\section{Acknowledgments}

This work was supported by NIH (DK-31842, DK20595, DK-02315, and DK-02742) and the General Clinical Research Center (M01 RR00055).

1. Kahn, B.B. 1998. Type 2 diabetes: when insulin secretion fails to compensate for insulin resistance. Cell. 92:593-596.

2. Coleman, D.L., and Hummel, K.P. 1973. The influence of genetic background on the expression of the obese $(\mathrm{Ob})$ gene in the mouse. Diabetologia. 9:287-293.

3. Polonsky, K.S., et al. 1988. Quantitative study of insulin secretion and clearance in normal and obese subjects. J. Clin. Invest. 81:435-441.

4. Jones, C.N.O., et al. 1997. Alterations in the glucose-stimulated insulin secretory dose-reponse curve and in insulin clearance in nondiabetic insulin-resistant individuals. J. Clin. Endocrinol. Metab. 82:1834-1838.

5. Kahn, S.E., et al. 1993. Quantification of the relationship between insulin sensitivity and $\beta$-cell function in human subjects. Evidence for a hyperbolic function. Diabetes. 42:1663-1672.

6. Pick, A., et al. 1998. Role of apoptosis in failure of $\beta$-cell mass compensation for insulin resistance and $\beta$-cell defects in the male Zucker diabetic fatty rat. Diabetes. 47:358-364.

7. Milburn, J.L., et al. 1995. Pancreatic $\beta$-cells in obesity. J. Biol. Chem. 270:1295-1299.

8. Becker, T.C., BeltrandelRio, H., Noel, R.J., Johnson, J.H., and Newgard, C.B. 1994. Overexpression of hexokinase I in isolated islets of Langerhans via recombinant adenovirus. Enhancement of glucose metabolism and insulin secretion at basal but not stimulatory glucose levels. J. Biol. Chem. 269:21234-21238.

9. Becker, T.C., et al. 1996. Differential effects of overexpressed glucokinase and hexokinase I in isolated islets. J. Biol. Chem. 271:390-394.

10. Cockburn, B.N., et al. 1997. Changes in pancreatic islet glucokinase and hexokinase activities with increasing age, obesity, and the onset of diabetes. Diabetes. 46:1434-1439.

11. Tokuyama, Y., et al. 1995. Evolution of $\beta$-cell dysfunction in the male Zucker diabetic fatty rat. Diabetes. 44:1447-1457.

12. Kadowaki, T. 2000. J. Clin. Invest. In press.

13. Withers, D.J., et al. 1998. Disruption of IRS- 2 causes type 2 diabetes in mice. Nature. 391:900-904.

14. Kido, Y., et al. 2000. Tissue-specific insulin resistance in mice with mutations in the insulin receptor, IRS-1, and IRS-2. J. Clin. Invest. 105:199-205.

15. Kulkarni, R.N., et al. 1999. Tissue-specific knockout of the insulin receptor in pancreatic $\beta$ cells creates an insulin secretory defect similar to that in type 2 diabetes. Cell. 96:329-339.

16. Kulkarni, R.N., et al. 1999. Altered function of insulin receptor substrate-1deficient mouse islets and cultured $\beta$-cell lines. J. Clin. Invest. 104:R69-R75.

17. Leibiger, I.B., Leibiger, B., Moede, T., and Berggren, P.O. 1998. Exocytosis of insulin promotes insulin gene transcription via the insulin receptor/PI-3 kinase/p70 s6 kinase and CaM kinase pathways. Mol. Cell. 1:933-938.

18. Xu, G.G., and Rothenberg, P.L. 1998. Insulin receptor signaling in the $\beta$ cell influences insulin gene expression and insulin content. Diabetes. 47:1243-1252.
19. Buchanan, T.A., Metzger, B.E., Frienkel, N., and Bergman, R.N. 1990. Insulin sensitivity and B-cell responsiveness to glucose during late pregnancy in lean and moderately obese women with normal glucose tolerance or mild gestational diabetes. Am. J. Obstet. Gynecol. 162:1008-1014.

20. Ryan, E.A., et al. 1995. Defects in insulin secretion and action in women with a history of gestational diabetes. Diabetes. 44:506-512.

21. Byrne, M.M., Sturis, J., Sobel, R.J., and Polonsky, K.S. 1996. Elevated plasma glucose $2 \mathrm{~h}$ postchallenge predicts defects in $\beta$-cell function. Am.J. Physiol. 270:E572-E579.

22. O’Meara, N.M., Sturis, J., Van Cauter, E., and Polonsky, K.S. 1993. Lack of control of ultradian insulin secretory oscillations in impaired glucose tolerance and in non-insulin-dependent diabetes mellitus. J. Clin. Invest. 92:262-267.

23. O'Meara, N., et al. 1993. Defects in $\beta$ cell function and insulin action in functional ovarian hyperandrogenism. J. Clin. Endocrinol. Metab. 76:1241-1247.

24. Ehrmann, D.A., et al. 1995. Insulin secretory defects in polycystic ovary syndrome: relationship to insulin sensitivity and family history of noninsulin dependent diabetes mellitus. J. Clin. Invest. 96:520-527.

25. Stern, M.P. 2000. Strategies and prospects for finding insulin resistance genes. J. Clin. Invest. 106:323-327.

26. Fajans, S., and Conn, J. 1954. An approach to the prediction of diabetes mellitus by modification of the glucose tolerance test with cortisone. Diabetes. 3:296-304.

27. Henriksen, J.E., Alford, F., Ward, G.M., and Beck-Nielsen, H. 1997. Risk and mechanism of dexamethasone-induced deterioration of glucose tolerance in non-diabetic first-degree relatives of NIDDM patients. Diabetologia. 40:1439-1448.

28. Cavaghan, M.K., Ehrmann, D.A., Byrne, M.M., and Polonsky, K.S. 1997. Treatment with the oral antidiabetic agent troglitazone improves $\beta$-cell responses to glucose in subjects with impaired glucose tolerance. J. Clin. Invest. 100:530-537.

29. Ehrmann, D.A., et al. 1997. Troglitazone improves defects in insulin action, insulin secretion, ovarian steroidogenesis, and fibrinolysis in women with polycystic ovary syndrome. J. Clin. Endocrinol. Metab. 82:2108-2116.

30. Boden, G., et al. 1991. Effects of fat on insulin-stimulated carbohydrate metabolism in normal men. J. Clin. Invest. 88:960-966.

31. Boden, G., and Jadali, F. 1991. Effects of lipid and basal carbohydrate metabolism in normal men. Diabetes. 40:686-692.

32. Shulman, G.I. 2000. Cellular mechanisms of insulin resistance. J. Clin. Invest. 106:171-176.

33. Crespin, S.R., Greenough, D.B., and Steinberg, D. 1973. Stimulation of insulin secretion by long-chain free fatty acids. J. Clin. Invest. 52:1979-1984.

34. Sako, Y., and Grill, V.E. 1990. A 48-hour lipid infusion in the rat timedependently inhibits glucose-induced insulin secretion and $\beta$-cell oxidation through a process likely coupled to fatty acid oxidation. Endocrinology. 127:1580-1589.

35. Stein, D.T., et al. 1996. Essentiality of circulating fatty acids for glucosestimulated insulin secretion in the fasted rat. J. Clin. Invest. 97:2728-2735.

36. Hirose, H., et al. 1996. Defective fatty acid-mediated $\beta$-cell compensation in Zucker diabetic fatty rats. J. Biol. Chem. 271:5633-5637.

37. Carpentier, A., et al. 1999. Acute enhancement of insulin secretion by FFA in humans is lost with prolonged FFA elevation. Am. J. Physiol. 276:E1055-E1066.

38. Cavaghan, M.K., Breda, E., Ehrmann, D.A., and Polonsky, K.S. 1999. Failure of $\beta$-cell compensation for insulin resistance induced by elevated free fatty acids in obese subjects. Diabetes. 48(Suppl. 1):A239. (Abstr.) 